\title{
Classification of String Theories via Topological Phases
}

\author{
Justin Kaidi®, ${ }^{1}$ Julio Parra-Martinez $\odot,{ }^{1}$ and Yuji Tachikawa ${ }^{2}$ \\ ${ }^{1}$ Mani L. Bhaumik Institute for Theoretical Physics, Department of Physics and Astronomy, University of California, \\ Los Angeles, California 90095, USA \\ ${ }^{2}$ Kavli Institute for the Physics and Mathematics of the Universe (WPI), University of Tokyo, Kashiwa, Chiba 277-8583, Japan
}

(Received 25 September 2019; accepted 20 February 2020; published 25 March 2020)

\begin{abstract}
We point out that the choice of phases in Gliozzi-Scherk-Olive projections can be accounted for by a choice of fermionic symmetry-protected topological phases on the world sheet of the string. This point of view not only easily explains why there are essentially two type II theories, but also predicts that there are unoriented type 0 theories labeled by $n \bmod 8$ and that there is an essentially unique choice of the type I world sheet theory. We also discuss the relationship between this point of view and the $K$-theoretic classification of D-branes.
\end{abstract}

DOI: 10.1103/PhysRevLett.124.121601

Introduction.-The most traditional method of studying superstring theory is via superstring perturbation theory. In the NS-R formalism, one starts with a world sheet theory which contains world sheet spinors which are spacetime vectors. Then, the theory is subjected to a process known as the Gliozzi-Scherk-Olive (GSO) projection [1,2]. It is well known that there are various possible choices of consistent GSO projections. In the standard textbook presentation, e.g., in [3], allowed GSO projections are determined by imposing various consistency conditions, such as the modular invariance of torus amplitudes. That the GSO projections give consistent results in higher genus amplitudes is not immediately clear in this presentation.

A formulation which works equally well for higher genera was found in [4]. There, it was pointed out that the GSO projection is a summation over the spin structure of the world sheet and that different GSO projections correspond to different possible phases assigned to spin structures in a way compatible with the cutting and the gluing of the world sheet. In particular, it was found there that the different signs appearing in type IIA and type IIB GSO projections are given by an invariant of the spin structure known as the Arf invariant. The Arf invariant is of order 2, which is closely related to the fact that there are only two type II theories.

Thanks to the developments initiated in condensed matter physics in the last decade, we now have a more physical understanding of this Arf invariant. Namely, it is the partition function of the low-energy limit of the $1+1 \mathrm{~d}$

Published by the American Physical Society under the terms of the Creative Commons Attribution 4.0 International license. Further distribution of this work must maintain attribution to the author(s) and the published article's title, journal citation, and DOI. Funded by SCOAP ${ }^{3}$. symmetry-protected topological (SPT) phase known as the Kitaev chain [5]. In general, the low-energy limit of a SPT phase is known as an invertible phase [6,7] and its partition function on a closed manifold is a phase (in the sense of a complex number of absolute value one) which behaves consistently under the cutting and gluing of the spacetime manifold. Conversely, it is now known that any such consistently assigned phase is given by the partition function of an invertible theory. Furthermore, there is now a general classification of possible invertible phases, or equivalently SPT phases, in terms of bordism groups [7-9].

This means that, with the technology currently available to us, we can now not only understand the consistency of a given GSO projection, but also enumerate all possible GSO projections. The aim of this Letter is to revisit known GSO projections from a modern viewpoint, and possibly find new ones. Note that we will restrict ourselves to projections which treat all fermions in the same manner-more general projections, which necessarily break spacetime Lorentz invariance, would be interesting to study.

For example, the Kitaev chain is known to be compatible with a parity transformation $\Omega$ such that $\Omega^{2}=(-1)^{F}$. Also, it is known that eight copies of the Kitaev chain protected by this symmetry are continuously connected to a completely trivial theory [10]. In this case, the partition function of the low-energy limit of the Kitaev chain is known as the Arf-Brown-Kervaire (ABK) invariant, and is of order 8. Now, let us consider an unoriented NS-R word sheet theory with $\Omega^{2}=(-1)^{F}$. Such a world sheet is said to have a pin $^{-}$ structure, which is a generalization of the concept of a spin structure to unoriented manifolds (see, e.g., Appendix A of [11] for an introduction). When we perform the GSO projection, or equivalently, when we sum over the pin $^{-}$ structures, we can now include $n$ copies of the ABK invariant. This leads to a series of unoriented type 0 string theories, labeled by $n$ mod 8 . Some of these theories have 
been discussed in the existing literature [12-21], but our unified description is new.

We can also ask whether it is possible to modify the GSO projection of the type I theory. In the type II theory, the leftand right-moving fermions couple to independent spin structures. This means that the world sheet fermions of the type I theory have neither $\mathrm{pin}^{+}$nor $\mathrm{pin}^{-}$structure; rather, one needs to consider the spin structure on the orientation double cover of the world sheet $[20,21]$. We will see below that there are nontrivial invertible phases for this structure, but that they will not lead to any genuinely new type I theory.

We also point out that our viewpoint provides a complementary way to understand the dependence of the $K$ theoretic classification of D-branes [22] on the choice of the GSO projection. For example, two type II theories differ by the presence of the Arf invariant or, equivalently, the Kitaev chain on the world sheet. Famously, the Kitaev chain has an unpaired fermionic zero mode on its boundary. This explains the fact that the boundary condition for the type IIA non-BPS D9-brane has an unpaired boundary fermion as originally observed in [23]. Mathematically, the presence of $n$ boundary fermions corresponds to the existence of the action of the Clifford algebra $\mathrm{Cl}(n)$, and the $K$ group $K^{n}(X)$ is defined in terms of unitary bundles with a specified action of $\mathrm{Cl}(n)$ [24]. With this observation, we see that the type IIB and type IIA theories have D-branes classified by $K^{0}(X)$ and $K^{1}(X)$, respectively.

Similarly, when we have $n$ copies of the ABK invariant, we have $n$ boundary fermions, leading to the existence of the action of the Clifford algebra $\mathrm{Cl}( \pm n)$. This means that the unoriented pin $^{-}$type 0 theory labeled by $n \bmod 8$ has D-branes classified by $K O^{+n}(X) \oplus K O^{-n}(X)$.

In the rest of the Letter, we will give more details on the points briefly summarized above. We will work in the lightcone gauge in the NS-R formulation. A longer version of this Letter, filling in many of the details, is forthcoming.

Type II strings. - Let us start with the type II string theory. We have eight left-moving fermions $\psi_{L}^{i}$ and eight right-moving fermions $\psi_{R}^{i}, i=1, \ldots, 8$. We allow for independent spin structures for the left and right movers. As the difference between two spin structures is a $\mathbb{Z}_{2}$ gauge field, we can equivalently say that we have a spin structure and a $\mathbb{Z}_{2}$ gauge field. The chiral GSO projection for the type II theories corresponds to the sum over the left- and right-moving spin structures on the world sheet.

In general, the global anomaly of a $D$-dimensional fermionic system with symmetry $G$ is controlled by $\mho_{\text {Spin }}^{D+1}(B G):=\operatorname{Hom}\left[\Omega_{D+1}^{\text {Spin }}(B G), U(1)\right]$, and the possible fermionic SPT phases with symmetry $G$ in $D$ dimensions are classified by $\mho_{\text {Spin }}^{D}(B G)$. Here, $\Omega_{d}^{\text {Spin }}(B G)$ is the bordism group of $d$-dimensional spin manifolds with a $G$ bundle, and $\widetilde{J}_{\text {Spin }}^{d}(B G)$ is its Pontryagin dual. $B G$ denotes the classifying space of $G$. The case relevant for us has $G=\mathbb{Z}_{2}$ and $D=2$,
TABLE I. Groups of SPT phases relevant to our analysis. The first four columns are classic [8,25]. The last column is new.

\begin{tabular}{cccccc}
\hline \hline$d$ & $\sigma_{\text {Spin }}^{d}(p t)$ & $\sigma_{\text {Spin }}^{d}\left(B \mathbb{Z}_{2}\right)$ & $\sigma_{\text {Pin }^{-}}^{d}(p t)$ & $\sigma_{\text {Pin }^{+}}^{d}(p t)$ & $\sigma_{\text {DPin }}^{d}(p t)$ \\
\hline 2 & $\mathbb{Z}_{2}$ & $\mathbb{Z}_{2}^{2}$ & $\mathbb{Z}_{8}$ & $\mathbb{Z}_{2}$ & $\mathbb{Z}_{2}^{2}$ \\
3 & 0 & $\mathbb{Z}_{8}$ & 0 & $\mathbb{Z}_{2}$ & $\mathbb{Z}_{8}$ \\
\hline \hline
\end{tabular}

for which the groups are given in Table I. (We note that, in the physics literature, our $\sigma_{\text {Spin }}^{d}(B G)$ is often denoted by $\Omega_{\text {Spin }}^{d}(B G)$, but this symbol signifies something different known as bordism cohomology for mathematicians, and the authors would like to avoid it.)

We see that the anomaly is characterized by $\mho_{\text {Spin }}^{3}\left(B \mathbb{Z}_{2}\right)=$ $\mathbb{Z}_{8}$. A single Majorana fermion coupled to two spin structures is known to have an anomaly which is a generator of this $\mathbb{Z}_{8}$, and, as pointed out in [26], the chiral GSO projection is nonanomalous thanks to the fact that we have $10-2=8$ Majorana fermions.

The SPT phases that we can add to the world sheet are classified by $\mho_{\text {Spin }}^{2}\left(B \mathbb{Z}_{2}\right)=\mathbb{Z}_{2}^{2}$, and their partition functions are given by

$$
(-1)^{n_{L} \operatorname{Arf}\left(\sigma_{L}\right)+n_{R} \operatorname{Arf}\left(\sigma_{R}\right)},
$$

where $\sigma_{L(R)}$ is the left(right)-moving spin structure, $\operatorname{Arf}(\sigma)$ is the mod-2 Arf invariant, and $n_{L(R)}=0,1$ label the four choices. As discussed above, they correspond to four distinct GSO projections possible in type II theories.

Then, we need to explain why we usually only talk about two type II theories. For this purpose, we recall that the Arf invariant is the low-energy limit of the Kitaev chain [8]. In the language of continuum field theory, this corresponds to the definition [11]

$$
e^{i \pi \operatorname{Arf}(\sigma)}=Z_{\text {ferm }}(m \gg 0, \sigma) / Z_{\text {ferm }}(m \ll 0, \sigma),
$$

where $Z_{\text {ferm }}(m, \sigma)$ is the partition function of a free massive Majorana fermion of mass $m$; both $\psi_{L, R}$ necessarily couple to the same spin structure $\sigma:=\sigma_{R}=\sigma_{L}$. The low-energy limit is taken by sending $|m| \rightarrow \infty$, and the denominator is the contribution from a Pauli-Villars regulator.

In fact this formula holds at finite mass

$$
e^{i \pi \operatorname{Arf}(\sigma)}=Z_{\text {ferm }}(+m, \sigma) / Z_{\text {ferm }}(-m, \sigma) .
$$

We further recall that the flip of the sign of the mass term, $m \rightarrow-m$, can be performed by $\left(\psi_{L}, \psi_{R}\right) \rightarrow\left(\psi_{L},-\psi_{R}\right)$. Taking the limit $m \rightarrow 0$, we find that a Majorana-Weyl fermion $\psi_{R}$ has an anomaly under $\psi_{R} \rightarrow-\psi_{R}$, and generates $(-1)^{\operatorname{Arf}\left(\sigma_{R}\right)}$.

This means that the parity transformation along a single spacetime direction, say $\left(\psi_{L}^{i=8}, \psi_{R}^{i=8}\right) \rightarrow\left(-\psi_{L}^{i=8},-\psi_{R}^{i=8}\right)$, produces $(-1)^{\operatorname{Arf}\left(\sigma_{L}\right)+\operatorname{Arf}\left(\sigma_{R}\right)}$, i.e., $n_{L}=n_{R}=1$ in (1). 
Therefore, there are only essentially two distinct type II GSO projections. The cases $\left(n_{L}, n_{R}\right)=(0,0),(1,1)$ are called type IIB and the cases $\left(n_{L}, n_{R}\right)=(0,1),(1,0)$ are called type IIA.

This also explains why $T$ duality exchanges type IIA and type IIB: $T$ duality along a spacetime direction implements $\left(\partial X_{R}, \psi_{R}\right) \rightarrow\left(-\partial X_{R},-\psi_{R}\right)$ while keeping $\left(\partial X_{L}, \psi_{L}\right)$ fixed. This generates $(-1)^{\operatorname{Arf}\left(\sigma_{R}\right)}$, thus, exchanging type IIB and type IIA.

We also note that the Kitaev chain has an unpaired Majorana fermion at its boundary. This explains the fact that the boundary condition for the type IIA D9-brane has one boundary fermion [23]. The fact that the type IIA Dbranes are classified by $K^{1}(X)$ while the type IIB D-branes are classified by $K^{0}(X)$ can also be explained from this point of view. We will discuss this in the context of unoriented pin $^{-}$type 0 strings below.

Oriented type 0 strings. - Next, let us consider oriented type 0 strings, obtained via diagonal GSO projections $[4,27]$. Here, we assign the same spin structure to both left- and right-moving fermions and, then, sum over this spin structure. The anomaly is controlled by $\mho_{\text {Spin }}^{3}(p t)$ and the invertible phases we can add on the world sheet are classified by $\mho_{\text {Spin }}^{2}(p t)$. Here, $p t$ means the absence of any additional symmetry.

A quick inspection of Table I shows that there is no anomaly to talk about, and there are two choices of the invertible phase, or equivalently, two choices of GSO projection. The invertible phases are simply given by $(-1)^{n \operatorname{Arf}(\sigma)}$ where $n=0,1$. The case $n=0$ is the oriented type $0 \mathrm{~B}$ string and the case $n=1$ is the oriented type $0 \mathrm{~A}$ string.

Compared to type II strings, there is a closed string tachyon in the NSNS sector, and the RR sector is doubled: the type 0B string has two $C$, two $C_{\mu \nu}$, and one nonchiral $C_{\mu \nu \rho \sigma}$ while the type 0A string has two $C_{\mu}$ and two $C_{\mu \nu \rho}$. Correspondingly, the D-brane spectra are also doubled, and are classified by $K^{0}(X) \oplus K^{0}(X)$ and $K^{1}(X) \oplus K^{1}(X)$, respectively.

Unoriented pin $^{-}$type 0 strings. - Now, let us move on to a discussion of unoriented versions of type 0 strings. On unoriented (Wick-rotated) $d$-dimensional spacetime, fermions transform under a double cover of $O(d)$. There are two distinct choices known as $\operatorname{Pin}^{ \pm}(d)$, distinguished by $\Omega^{2}=( \pm 1)^{F}$ where $\Omega$ is a lift of the parity transformation along a single direction. In our context, this distinction manifests itself, for example, in the spin structure along the boundary of a Möbius strip. Going around this boundary is homotopically equivalent to going twice around the unorientable cycle around the cross cap and, thus, involves $\Omega^{2}$. Therefore, it is automatically in the NS sector with pin $^{-}$ structure, and in the $\mathrm{R}$ sector with $\mathrm{pin}^{+}$structure.

First, we discuss the $\mathrm{pin}^{-}$case. The system is automatically anomaly free since $\mho_{\mathrm{Pin}^{-}}^{3}(p t)=0$. Then, we have the choice of invertible phases on the world sheet, given by $\mho_{\text {Pin }^{-}}^{2}(p t)=\mathbb{Z}_{8}$. These invertible phases have been studied previously in $[8,28,29]$, and in the condensed matter literature in [10,30,31]. The partition functions are given by

$$
e^{(2 \pi i / 8) n \mathrm{ABK}(\sigma)},
$$

where $n$ is an integer modulo 8 , and we have $\operatorname{ABK}(\sigma)=$ \pm 1 on $\mathbb{R} \mathbb{P}^{2}$. The ABK invariant admits a physical definition in terms of massive fermion partition functions, of the same form as for the Arf invariant (2) but for a system with time-reversal symmetry. As a result of the Dai-Freed theorem [32], it can also be expressed naturally in terms of the $\eta$-invariant familiar from index theory [11]. Note that, with $n=4, e^{(2 \pi i / 8) n \mathrm{ABK}(\sigma)}$ assigns the phase -1 to $\mathbb{R P}^{2}$ and, thus, maps $\mathrm{O}^{-}$to $\mathrm{O}^{+}$. More mathematically, this means that

$$
e^{(2 \pi i / 8) 4 \mathrm{ABK}(\sigma)}=(-1)^{\int w_{1}^{2}},
$$

where $w_{1}$ is the first Stiefel-Whitney class of the manifold, measuring the nonorientability. Theories differing by four copies of ABK differ by this same phase in the partition function.

We also note the following: on oriented surfaces, $\operatorname{ABK}(\sigma)=4 \operatorname{Arf}(\sigma)$ modulo 8, which means that the cases $n=0,2,4,6$ are orientifolds of type $0 \mathrm{~B}$ and the cases $n=1,3,5,7$ are of type $0 \mathrm{~A}$. The fact that type $0 \mathrm{~A}$ and $0 \mathrm{~B}$ theories split into four cases each when we consider unoriented theories was also mentioned in Appendix F of a recent paper [33].

The Klein bottle admits four pin ${ }^{-}$structures, and is obtained by gluing together two copies of $\mathbb{R} \mathbb{P}^{2}$. As such, the ABK invariants are $( \pm 1)+( \pm 1)=-2,0,0,+2$. The Klein bottle amplitude is a trace of $\Omega$ on the closed-string Hilbert space, and the cases where $\operatorname{ABK}(\sigma)= \pm 2$ correspond to the trace of $\Omega$ in the RR sector, while those with $\operatorname{ABK}(\sigma)=0$ correspond to the trace in the NSNS sector. Therefore, including the phase (4) in the GSO projection only modifies the action of $\Omega$ in the RR sector, and we have

$$
\Omega_{\mathrm{RR}}^{(n)}=\Omega_{\mathrm{RR}}^{(0)} e^{(2 \pi i / 4) n} .
$$

We find that the NSNS sector contains the tachyon, the metric, and the dilaton independent of $n$. In the RR sector, one has

$$
\begin{array}{llll}
C_{\mu \nu}, & C_{\mu \nu}^{\prime} & & (n=0,4) ; \\
C_{\mu}, & C_{\mu \nu \rho} & & (n=1,5) ; \\
C, & C^{\prime}, & C_{\mu \nu \rho \sigma} & (n=2,6) ; \\
C_{\mu}, & C_{\mu \nu \rho} & & (n=3,7),
\end{array}
$$

where $C_{\mu \nu \rho \sigma}$ is a nonchiral four form. Correspondingly, we can find the following nontorsion D-branes using the standard boundary state formalism 
$D 1, D 1^{\prime}, D 5, D 5^{\prime}, D 9, D 9^{\prime}$

$(n=0,4)$;

$D 0, D 2, D 4, D 6, D 8$

$(n=1,5)$;

$D(-1), D(-1)^{\prime}, D 3, D 3^{\prime}, D 7, D 7^{\prime}$

$(n=2,6)$;

$D 0, D 2, D 4, D 6, D 8$

$(n=3,7)$.

These nontorsion D-branes match the $K$-theoretic classification given by $K O^{+n}(X) \oplus K O^{-n}(X)$. This $K$-theoretic classification of $\mathrm{D}$-branes can also be interpreted as follows.

Let us consider a system of $n$ copies of the Kitaev chain. When put on a segment, it has $n$ fermion zero modes $\psi_{L}^{a}$ on the left boundary and another $n$ fermion zero mode $\psi_{R}^{a}$ on the right boundary, $a=1, \ldots, n$. When we assign the $T$ transformation $\mathrm{T} \psi_{L}^{a}=+\psi_{L}^{a}$ and $\mathrm{T} \psi_{R}^{a}=+\psi_{R}^{a}$, the anticommutators are $\left\{\psi_{L}^{a}, \psi_{L}^{b}\right\}=+2 \delta^{a b}$ and $\left\{\psi_{R}^{a}, \psi_{R}^{b}\right\}=$ $-2 \delta^{a b}[10,11]$. The algebra $\left\{\psi^{a}, \psi^{b}\right\}= \pm \delta^{a b}$ with $a, b=$ $1, \ldots, n$ is known as the Clifford algebra $\mathrm{Cl}( \pm n)$. This means that, when $n$ copies of the ABK invariant are introduced, there are two types of boundaries, one carrying the action of $\mathrm{Cl}(+n)$ and another carrying the action of $\mathrm{Cl}(-n)$. Now, the group $K O^{n}(X)$ is defined in terms of orthogonal bundles with an additional action of $\mathrm{Cl}(n)$ [24]. Therefore, the $\mathrm{D}$-branes in the unoriented $\mathrm{pin}^{-}$ type 0 string specified by $n \bmod 8$ are classified by $K O^{+n}(X) \oplus K O^{-n}(X)$.

Unoriented $\mathrm{pin}^{+}$type 0 strings. - Let us briefly mention the $\mathrm{pin}^{+}$case, which has been studied previously under the name type 0' strings $[19,34,35]$. Here, the anomaly is characterized by $\widetilde{J}_{\mathrm{Pin}^{+}}^{3}(p t)=\mathbb{Z}_{2}$. As we have eight fermions, the GSO projection is anomaly free. Then, the invertible phases on the world sheet are classified by $\mho_{\mathrm{Pin}^{+}}^{2}(p t)=\mathbb{Z}_{2}$. It is known that the invertible phases are given by

$$
(-1)^{n \operatorname{Arf}(\hat{\sigma})}, \quad n=0,1,
$$

where $\hat{\sigma}$ is the spin structure of the orientation double cover of the world sheet. When orientable, $\operatorname{Arf}(\hat{\sigma})=$ $\operatorname{Arf}\left(\sigma_{L}\right)+\operatorname{Arf}\left(\sigma_{R}\right)$. This can be generated by the spacetime parity transformation along a single direction as we already discussed, and therefore, there is only an essentially unique way to perform the GSO projection. This GSO projection removes the closed string tachyon and has an interesting Green-Schwarz cancellation, but we do not have anything to add to the discussions given in the references cited above.

Type I string.-Now, we study the possibility of a new GSO projection for the type I string. It will turn out that there is no new possibility.

In order to explain this, we need to study the spin structure of the orientation double cover. Our main interest here is the world sheet, which is two dimensional, but to formulate the bordism group it is useful to work in arbitrary dimensions. Let us, then, consider (Wick-rotated) $n$-dimensional manifolds. When the manifold is oriented, the spin structure of the orientation double cover corresponds to considering $\operatorname{Spin}(n) \times \mathbb{Z}_{2}$, which is a double cover of $\mathrm{SO}(n) \times \mathbb{Z}_{2}$. Therefore, when the manifold is unoriented, we need to consider a double cover

$$
0 \rightarrow \mathbb{Z}_{2} \rightarrow G \rightarrow \mathrm{O}(n) \times \mathbb{Z}_{2} \rightarrow 0 .
$$

The precise extension is specified by an element of $H^{2}\left(B \mathrm{O}(n) \times B \mathbb{Z}_{2}, \mathbb{Z}_{2}\right)$ and is given by $w_{2}+w_{1}^{2}+w_{1} a$, where $w_{1,2}$ are the usual Stiefel-Whitney classes of $\mathrm{O}(n)$ and $a$ is the generator of $H^{1}\left(B \mathbb{Z}_{2}, \mathbb{Z}_{2}\right)=\mathbb{Z}_{2}$. We can check that this group $G$ contains $\operatorname{Spin}(n) \times \mathbb{Z}_{2}$ as it should, and also contains both $\operatorname{Pin}^{+}(n)$ and $\operatorname{Pin}^{-}(n)$. This is, in some sense, a double Pin group, and we denote it by $G=\operatorname{DPin}(n)$.

We need to find $\mho_{\mathrm{DPin}}^{2,3}(p t)$. This can be computed using the Atiyah-Hirzebruch spectral sequence for twisted spin bordism groups, using the information on the differential $d_{2}$ recently found in [36]. The result is shown in Table I; we will detail the computations in the upcoming full paper. The GSO projection is anomaly free, since $\mho_{\text {DPin }}^{3}(p t)=\mathbb{Z}_{8}$, and we have eight fermions. The invertible phases on the world sheet are classified by $\mho_{\text {DPin }}^{2}(p t)=\mathbb{Z}_{2}^{2}$, whose generators are simply

$$
(-1)^{\int w_{1}^{2}}, \quad(-1)^{\operatorname{Arf}(\hat{\sigma})}
$$

We already noted in (5) that $(-1)^{\int w_{1}^{2}}$ simply exchanges O9 ${ }^{ \pm}$. We also already saw that $(-1)^{\operatorname{Arf}(\hat{\sigma})}$ can be generated by a spacetime parity transformation along a single direction and, therefore, does not lead to an essentially different GSO projection. Hence, one obtains only the usual type I and I strings.

Conclusion.-Topological phases first developed in condensed matter physics have come to play an increasingly prominent role in the study of the dynamics of continuum quantum field theory, largely through their connection to anomalies. In this Letter, we have shown that the recent insights into such phases can also be connected to traditional results about the classification of string world sheet theories. Looking forward, we expect that this connection will prove useful to the study of other aspects of string theory, including orbifolds, heterotic strings, and compactifications. Of particular interest would be understanding the implications of these methods for string model building. We hope to explore some of these applications in the future. We also hope that this fruitful interplay between condensed matter and string theory will be taken in the other direction, using traditional string results to shed light on condensed matter systems. 
The authors thank the TASI 2019 summer school at University of Colorado, Boulder, for providing an ideal environment for collaboration. The authors also thank Arun Debray, Ryohei Kobayashi, and Ryan Thorngren for their help in computing the relevant bordism groups, and Max Metlitski for many useful discussions. Y. T. is, in part, supported by World Premier International Initiative, MEXT, Japan at IPMU, the University of Tokyo, and, in part, by JSPS KAKENHI Grant-in-Aid (Wakate-A) No. $17 \mathrm{H} 04837$ and JSPS KAKENHI Grant-in-Aid (Kiban-S) No. 16H06335. J.P.M. is supported by the U.S. Department of State through a Fulbright scholarship. J. K. and J. P. M. thank the Mani L. Bhaumik Institute for generous support.

Note added.-While this Letter was close to completion, the authors were informed that E. Witten has an unpublished work with large overlap with this work; two seminars he gave can be found in $[37,38]$. The authors thank Kantaro Ohmori and Matthew Heydeman for this information.

[1] F. Gliozzi, J. Scherk, and D. I. Olive, Supergravity and the spinor dual model, Phys. Lett. 65B, 282 (1976).

[2] F. Gliozzi, J. Scherk, and D. I. Olive, Supersymmetry, supergravity theories and the dual spinor model, Nucl. Phys. B122, 253 (1977).

[3] J. Polchinski, String Theory. Vol. 2: Superstring Theory and Beyond, Cambridge Monographs on Mathematical Physics (Cambridge University Press, Cambridge, 2007).

[4] N. Seiberg and E. Witten, Spin structures in string theory, Nucl. Phys. B276, 272 (1986).

[5] A. Y. Kitaev, Unpaired Majorana fermions in quantum wires, Phys. Usp. 44, 131 (2001).

[6] D. S. Freed, Short-range entanglement and invertible field theories, arXiv:1406.7278.

[7] D. S. Freed and M. J. Hopkins, Reflection positivity and invertible topological phases, arXiv:1604.06527.

[8] A. Kapustin, R. Thorngren, A. Turzillo, and Z. Wang, Fermionic symmetry protected topological phases and cobordisms, J. High Energy Phys. 12 (2015) 052.

[9] K. Yonekura, On the cobordism classification of symmetry protected topological phases, Commun. Math. Phys. 368, 1121 (2019).

[10] L. Fidkowski and A. Kitaev, The effects of interactions on the topological classification of free fermion systems, Phys. Rev. B 81, 134509 (2010).

[11] E. Witten, Fermion path integrals and topological phases, Rev. Mod. Phys. 88, 035001 (2016).

[12] M. Bianchi and A. Sagnotti, On the systematics of open string theories, Phys. Lett. B 247, 517 (1990).

[13] A. Sagnotti, Some properties of open string theories, in Supersymmetry and Unification of Fundamental Interactions. Proceedings, International Workshop, SUSY 95, Palaiseau, France, 1995 (1995), pp. 473-484.
[14] A. Sagnotti, Surprises in open string perturbation theory, Nucl. Phys. B, Proc. Suppl. 56, 332 (1997).

[15] R. Blumenhagen, A. Font, and D. Lust, Nonsupersymmetric gauge theories from D-branes in type 0 string theory, Nucl. Phys. B560, 66 (1999).

[16] R. Blumenhagen, A. Font, A. Kumar, and D. Lust, Aspects of type 0 string theory, Classical Quantum Gravity 17, 989 (2000).

[17] R. Blumenhagen and A. Kumar, A Note on orientifolds and dualities of type OB string theory, Phys. Lett. B 464, 46 (1999).

[18] I. R. Klebanov, N. A. Nekrasov, and S. L. Shatashvili, An Orbifold of type OB strings and nonsupersymmetric gauge theories, Nucl. Phys. B591, 26 (2000).

[19] O. Bergman and M. R. Gaberdiel, Dualities of type 0 strings, J. High Energy Phys. 07 (1999) 022.

[20] J. Distler, D. S. Freed, and G. W. Moore, Orientifold precis, in Mathematical Foundations of Quantum Field theory and Perturbative String Theory, edited by H. Sati and U. Schreiber (American Mathematical Society, Providence, R.I., 2011).

[21] J. Distler, D. S. Freed, and G. W. Moore, Spin structures and superstrings, arXiv:1007.4581.

[22] E. Witten, D-branes, and K theory, J. High Energy Phys. 12 (1998) 019.

[23] P. Horava, Type IIA D-branes, K theory, and matrix theory, Adv. Theor. Math. Phys. 2, 1373 (1998).

[24] M. Karoubi, in K-Theory: An Introduction, Classics in Mathematics (Springer Berlin Heidelberg, Berlin, Heidelberg, 2008).

[25] R. C. Kirby and L. R. Taylor, Pin structures on lowdimensional manifolds, in Geometry of Low-Dimensional Manifolds, Vol. 151 of London Mathematical Society Lecture Note Series Vol. 2 (1990), pp. 177-242.

[26] Y. Tachikawa and K. Yonekura, Why are fractional charges of orientifolds compatible with Dirac quantization?, SciPost Phys. 7, 058 (2019).

[27] L. J. Dixon and J. A. Harvey, String theories in tendimensions without space-time supersymmetry, Nucl. Phys. B274, 93 (1986).

[28] A. Debray and S. Gunningham, The Arf-Brown TQFT of pin $^{-}$surfaces, in Topology and Quantum Theory in Interacti (American Mathematical Society, Providence, R.I., 2018).

[29] A. Turzillo, Diagrammatic state sums for 2D pin-minus TQFTs, arXiv:1811.12654.

[30] G. Y. Cho, K. Shiozaki, S. Ryu, and A. W. W. Ludwig, Relationship between symmetry protected topological phases and boundary conformal field theories via the entanglement spectrum, J. Phys. A 50, 304002 (2017).

[31] K. Shiozaki, H. Shapourian, and S. Ryu, Manybody topological invariants in fermionic symmetryprotected topological phases, Phys. Rev. B 95, 205139 (2017).

[32] X.-z. Dai and D. S. Freed, Eta invariants and determinant lines, J. Math. Phys. (N.Y.) 35, 5155 (1994); Erratum, J. Math. Phys. (N.Y.) 42, 2343 (2001).

[33] D. Stanford and E. Witten, JT gravity and the ensembles of random matrix theory, arXiv:1907.03363. 
[34] R. Blumenhagen, A. Font, and D. Lust, Tachyon free orientifolds of type 0B strings in various dimensions, Nucl. Phys. B558, 159 (1999).

[35] C. Angelantonj and A. Armoni, Nontachyonic type 0B orientifolds, nonsupersymmetric gauge theories and cosmological RG flow, Nucl. Phys. B578, 239 (2000).

[36] R. Thorngren, Anomalies and bosonization, arXiv:1810 .04414
[37] E. Witten, Anomalies and nonsupersymmetric d-branes, in Proceedings at SCGP, 2018, https://www.youtube.com/ watch?v=SzzQ9jecuT4.

[38] E. Witten, Nonsupersymmetric d-branes and the Kitaev fermion chain, in Proceedings at the Shoucheng Zhang Memorial Workshop, 2019, https://www.youtube.com/ watch?v=P_JoLg_NkrA. 